J. Austral. Math. Soc. 19 (Series B), (1975), 165-172.

\title{
THE EFFECT OF VISCOUS DISSIPATION ON NON-LINEAR CONVECTION AT HIGH RAYLEIGH NUMBER
}

\author{
R. VAN DER BORGHT
}

(Received 17 September 1975)

\begin{abstract}
When studying deep convection in a compressible medium the effects of viscous dissipation can become important and must be taken into account in any realistic model. But even in shallow convection, for which the Boussinesq approximation is valid, the viscous dissipation effects will become important at high Rayleigh numbers. These effects are estimated with the help of asymptotic methods and the results are compared with those obtained by numerical integration.
\end{abstract}

\section{Introduction}

In a linear analysis one can neglect the viscous dissipation term in the energy equation because it is of the second order in the velocities. It is common procedure to neglect such terms in a non-linear analysis of convection although some of the numerical integrations have been carried out to very high values of the Rayleigh number and corresponding high values of the velocities. The relative importance of the viscous dissipation term is governed by a parameter of the form $V R=g \alpha d / C_{v}$ which, in the Boussinesq approximation, can be written $V R=g d /\left(C_{p} T\right)$. The factor $g / C_{p}$ is the adiabatic temperature gradient and $\left[g /\left(C_{p} T\right)\right]^{-1}$ is therefore the temperature scale height $H_{T}$. It follows that the parameter $V R=d / H_{T}$. If $d \gg H_{T}$ the influence of the viscous dissipation can be quite large and as shown by Turcotte et al. [4] the viscous dissipation has an inhibiting effect on convection and can, in extreme cases, stop convection altogether. Unfortunately the effects of the pressure variations were not considered and the computations should be repeated within the framework of an anelastic model. This should certainly be carried out if one wants to investigate deep convection in the outer layers of stars, for example, in a study of the supergranulation, since the temperature can change by a factor of ten over the top $8000 \mathrm{~km}$ of the convective layer. 
Fortunately, in applications to convection in the earth's atmosphere, the earth's outer mantle and even the outer layers of the sun (to a depth of 300 $\mathrm{km})$ the parameter $V R=g d /\left(C_{p} T\right)$ is much smaller than one and the application of the Boussinesq approximation is well justified.

The effect of dissipative heating on convective flows have recently been studied by Hewitt et al. [1] who have derived an approximate expression for the ratio of dissipative heating to the conductive heat flux. It is our purpose to study in this paper the effects of viscous dissipation on the flow characteristics in convection cells, at high Rayleigh number, and to compare these results with those obtained by numerical integrations of the basic equations.

\section{Basic equations}

The basic equations of the problem have been derived elsewhere and these can be reduced to the following form in the case of incompressible fluids (Van der Borght [5])

$$
\begin{aligned}
\left(D^{2}-a^{2}\right)^{2} W= & R a^{2} F+\frac{C}{\sigma}\left\{W D\left(D^{2}-a^{2}\right) W+2 D W\left(D^{2}-a^{2}\right) W\right\} \\
D^{2} T_{0}= & D(F W)-V\left\{4(D W)^{2}+\left(W+\frac{D^{2} W}{a^{2}}\right)^{2} a^{2}\right\} \\
\left(D^{2}-a^{2}\right) F= & W D T_{0}+C(F D W+2 W D F) \\
& -V C\left\{5(D W)^{2}+a^{2}\left(W+\frac{D^{2} W}{a^{2}}\right)^{2}\right\}
\end{aligned}
$$

where $D \equiv \partial / \partial z$.

The velocity and temperature distributions within the fluid are given by the following expressions

$$
u=\left(\frac{D W}{a^{2}} \frac{\partial f}{\partial x}, \frac{D W}{a^{2}} \frac{\partial f}{\partial y}, W f\right)
$$

and

$$
T=T_{0}+F
$$

where $W, T_{0}$ and $F$ are functions of $z$ to be obtained from the integration of the above differential equations, subject to the following boundary conditions:

and

$$
\begin{aligned}
& W=0 \quad \text { at } \quad z=0 \quad \text { and } \quad z=1 \\
& D^{2} W=0 \quad \text { at } \quad z=0 \text { and } z=1 \\
& F=0 \quad \text { at } \quad z=0 \quad \text { and } \quad z=1 \\
& T_{0}=0 \quad \text { at } \quad z=0 \\
& T_{0}=-1 \text { at } z=1 \text {. }
\end{aligned}
$$


The function $f$ which appears in expression (4) is a solution of the following differential equation

$$
\frac{\partial^{2} f}{\partial x^{2}}+\frac{\partial^{2} f}{\partial y^{2}}=-a^{2} f
$$

and its actual functional form depends on the assumed planform of the convection cells. For hexagonal cells $C=1 / \sqrt{6}$; in this paper we shall restrict ourselves to rolls and convective cells with square and rectangular planforms for which $C=0$.

Other parameters appearing in the basic equations are defined as follows:

$$
\begin{aligned}
& \sigma=\nu / \kappa=\text { Prandtl number } \\
& R=\frac{g \alpha d^{3} \Delta T}{\kappa \nu}=\text { Rayleigh number } \\
& V=\frac{\nu \kappa}{d^{2} C_{v} \Delta T}
\end{aligned}
$$

Terms derived from the inclusion of viscous dissipation in the basic equations are identified by this last factor.

If we use the Boussinesq approximation the coefficient of volume expansion $\alpha$ and specific heat at constant volume $C_{v}$ are replaced by $1 / T_{n}$ and the specific heat at constant pressure $C_{p}$ respectively; $T_{n}$ is the temperature at some point within the layer.

The important controlling parameter is then

$$
V R=g d /\left(C_{p} T_{n}\right) .
$$

In the earth's upper mantle $V R \simeq 0.117$ (Hewitt et al. [1]) whereas in the outer layers of the sun $V R \simeq 0.162$. To arrive at this last value we have adopted $\alpha=2 \times 10^{-4}{ }^{\circ} K^{-1}, d=3 \times 10^{7} \mathrm{~cm}, g=2.7 \times 10^{4} \mathrm{~cm} \mathrm{sec}^{-2}$ (Ledoux et al. [3]) and $C_{p}=10^{9} \mathrm{~cm}^{2} \sec ^{-2 \circ} K^{-1}$ (Waters [8]).

The numerical integrations have been carried out for $V R=0.25$.

\section{Comparison between analytic and numerical solutions}

Introducing a new variable $\bar{W}$ defined as follows

$$
W=\sqrt{R a^{2} N} \bar{W}
$$

where $N$ is the Nusselt number corresponding to the case where the viscous dissipation is neglected, this new variable satisfies the equation 


$$
\left(D^{2}-a^{2}\right)^{2} \bar{W}=\frac{1}{\bar{W}}+\frac{(V R) a^{2}}{\bar{W}} \int_{0}^{2}\left[4(D \bar{W})^{2}+\left(\bar{W}+\frac{D^{2} \bar{W}}{a^{2}}\right)^{2} a^{2}\right] d z .
$$

This reduces to the usual expression (e.g. Van der Borght and Murphy 1973) when $V R=0$, i.e. when the effects of viscous dissipation are neglected.

Applying an expansion of the form

$$
\bar{W}=W_{1} \sin \pi z+W_{2} \sin 2 \pi z
$$

one gets, after some very lengthy but straightforward algebra,

$$
\frac{1}{W_{1}^{2}}=\frac{1}{2}\left(\pi^{2}+a^{2}\right)^{2}-4 V R\left\{b_{1} / 2+c_{1} W_{2} / W_{1}\right\}
$$

and

$$
\frac{W_{2}}{W_{1}}=\frac{16(V R)\left\{-2 b_{1} / \pi^{2}+4 b_{4} / 3 \pi\right\}}{\left(4 \pi^{2}+a^{2}\right)^{2}+2\left(\pi^{2}+a^{2}\right)^{2}}
$$

with

$$
\begin{aligned}
& b_{1}=\frac{a^{2} \pi^{2}}{2}+\frac{\left(a^{2}-\pi^{2}\right)^{2}}{8} \\
& b_{4}=\frac{a^{2} \pi}{4}-\frac{1}{16 \pi}\left(a^{2}-\pi^{2}\right)^{2} \\
& c_{1}=\frac{4 b_{1}}{\pi^{2}}-\frac{8 \pi}{3} b_{4}+\frac{2 b_{3}}{\pi}+\frac{2 b_{5}}{3 \pi}
\end{aligned}
$$

where

$$
\begin{aligned}
& b_{3}=2 \pi a^{2}+\frac{1}{4 \pi}\left(a^{4}+4 \pi^{4}-5 a^{2} \pi^{2}\right) \\
& b_{5}=\frac{2}{3} \pi a^{2}-\frac{1}{12 \pi}\left(a^{4}+4 \pi^{4}-5 a^{2} \pi^{2}\right) .
\end{aligned}
$$

If we adopt a horizontal wave number $a=\pi / \sqrt{2}$, which corresponds to the critical value of the Rayleigh number, it follows from the previous expressions that

$$
\frac{W_{2}}{W_{1}}=-6.8223 \times 10^{-3}
$$

This shows that the effect of the inclusion of viscous dissipation will result in a slight asymmetry of the velocity curve, with the maximum displaced towards the upper part of the convective layer.

A comparison with the results of numerical integrations at high Rayleigh number $\left(R=1.121 \times 10^{8}\right)$ shows that the asymmetry given in (17) is of the 
right order of magnitude. The inclusion of higher order terms in the expression (13) would lead to a closer approximation to the value of $-8.688 \times 10^{-3}$ obtained by numerical methods. The amount of algebraic manipulations which would be required is prohibitive and is not warranted by the small order of the effect.

The Nusselt number $N$, corresponding to the case when the viscous dissipation is neglected, can be determined using a method first introduced by Howard [2] and it can be shown that

$$
N=(2.124)^{-4 / 3} k_{0}^{2 / 3}\left(a^{2} R\right)^{1 / 3}
$$

where

$$
\begin{gathered}
k_{0}=\pi A_{1}\left(1+3 A_{3} / A_{1}\right) \\
A_{1}^{2}=\frac{2}{\left(\pi^{2}+a^{2}\right)^{2}}\left(1-\frac{\left(\pi^{2}+a^{2}\right)^{2}}{\left(9 \pi^{2}+a^{2}\right)^{2}}\right)
\end{gathered}
$$

and

$$
\frac{A_{3}}{A_{1}}=\frac{\left(\pi^{2}+a^{2}\right)^{2}}{\left(9 \pi^{2}+a^{2}\right)^{2}}
$$

The value of $N$, as given by (18), represents the Nusselt number for extremely large values of the Rayleigh number. For $a=\pi / \sqrt{2}$ and $R=1.121 \times 10^{8}$ this gives

$$
N=141.4087
$$

which is somewhat larger than the computed value of 140.6333 .

As we have seen the inclusion of the viscous dissipation term in the equations has only a small effect on the distribution of the vertical velocity $W$; the same applies to the temperature fluctuation $F$. If one compares the distribution of the average temperature in the two cases one sees that there is a more marked effect, especially if the parameter $V R$ is of the order of 0.25 .

When the viscous dissipation is taken into account the temperature gradients $D T_{0}$ at the upper and lower boundaries are no longer equal and we denote the absolute values of these gradients by $N_{u}$ and $N_{b}$ respectively.

The average temperature at the midpoint is given by the expression

$T_{0}(1 / 2)=\int_{0}^{1 / 2} F W d z-V \int_{0}^{1 / 2} d z \int_{0}^{z}\left\{4(D W)^{2}+\left(W+\frac{D^{2} W}{a^{2}}\right)^{2} a^{2}\right\} d z-\frac{N_{b}}{2} ;$

keeping in mind that $W_{2} / W_{1} \leqslant 1$, it follows after some lengthy transformations and integrations that 


$$
T_{0}(1 / 2)=-0.5+16(V R)^{2} a^{2} N W_{1} W_{2}\left\{\frac{8}{3}+\frac{1}{6 \pi^{2} a^{2}}\left[a^{4}-5 \pi^{2} a^{2}+2 \pi^{4}\right]\right\} .
$$

For $a=\pi / \sqrt{2}$ this gives a value of $T(1 / 2)=-0.4218$ which can be compared with the computed value of -0.442 .

The magnitude of the average temperature gradient $N_{b}=\left(D T_{0}\right)$ at the bottom of the convective layer is given by the following expression

$$
N_{b}=1+\int_{0}^{1} F W d z-V \int_{0}^{1} d z \int_{0}^{z}\left\{4(D W)^{2}+\left(W+\frac{D^{2} W}{a^{2}}\right)^{2} a^{2}\right\} d z
$$

from which it follows that

$$
\frac{N_{u}-N_{b}}{N}=2 V R a^{2} \int_{0}^{1} d z \int_{0}^{z}\left\{4(D \bar{W})^{2}+\left(\bar{W}+\frac{D^{2} \bar{W}}{a^{2}}\right)^{2} a^{2}\right\} d z .
$$

Substituting the expansion for $\bar{W}$ in this equation one gets after a few integrations

$$
\Delta=\frac{N_{u}-N_{b}}{N}=V R+32(V R)^{2} a^{2}\left(W_{1} W_{2}\right)\left\{\frac{8}{3}+\frac{B}{\pi^{2}}\right\}
$$

where

$$
B=\frac{1}{2 a^{2}}\left[a^{4}-5 \pi^{2} a^{2}+2 \pi^{4}\right]
$$

It is seen from this expression that, for $V R \ll 1$, the ratio $\Delta$ is independent of the horizontal wave number. For higher values of $V R$ the higher order terms would have to be taken into account. At large Rayleigh number, for example $1.121 \times 10^{8}$, and for $V R=0.25$ the numerical integrations yield a value of $\Delta=0.2482$. This is in very good agreement with the value predicted from equation (27) for which $\Delta=0.2489$.

The parameter $\Delta$ can be defined as the ratio of the internally generated heat $\Phi$ to the average heat flux $\mathscr{F}$. The ratio of $\Phi$ to the heat flux through the upper boundary $\mathscr{F}_{u}$ can be calculated and one finds that for large $R$

$$
\Phi / \mathscr{F}_{u}=0.22
$$

and is lower than the one predicted by expression (27).

It is also seen from formula (27) that the ratio $\Delta$ is independent of the Rayleigh number. This result is of course derived from an asymptotic theory and only applies at high Rayleigh number. Table 1 shows the variation of $\Delta$ with Rayleigh number. 
TABLE 1

Variation of the ratio $\Delta$ of internally generated heat to the average heat flux with Rayleigh number $R$

\begin{tabular}{|c|c|c|c|}
\hline$R$ & $\Delta$ & $R$ & $\Delta$ \\
\hline 657.5 & 0.0000 & 1953.0 & 0.1589 \\
\hline 700.0 & 0.0273 & 3906.0 & 0.1868 \\
\hline 800.0 & 0.0674 & 7813.0 & 0.2011 \\
\hline 900.0 & 0.0910 & $1.563 \times 10^{4}$ & 0.2125 \\
\hline 1000.0 & 0.1067 & $1.25 \times 10^{5}$ & 0.2321 \\
\hline 1200.0 & 0.1267 & $1.0 \times 10^{6}$ & 0.2410 \\
\hline 1400.0 & 0.1393 & $1.121 \times 10^{8}$ & 0.2478 \\
\hline
\end{tabular}

It follows from equations (13) and (14) that

$$
W_{1} W_{2}=\frac{2}{\left(\pi^{2}+a^{2}\right)^{2}} \times \frac{V R\left[-\frac{2 b_{1}}{\pi^{2}}+\frac{4 b_{4}}{3 \pi}\right]}{\left(4 \pi^{2}+a^{2}\right)^{2}+2\left(\pi^{2}+a^{2}\right)^{2}}
$$

is of the order of $V R$. The second term in expression (27) for $\Delta$ is therefore of the third order in this quantity and consequently quite small. For $V R=0.25$ and $a=\pi / \sqrt{2}$ this term is negative and of the order of $10^{-3}$.

For a given value of the Rayleigh number, for example $R=1.121 \times 10^{8}$, one would expect $\Delta$ to tend to zero as $a$ is either increasing or decreasing. Zero values of $\Delta$ would occur for values of $a_{1}$ and $a_{2}$ corresponding to the points of intersection of the line $R=$ constant with the neutral stability curve. Nevertheless the results of this paper indicate that these changes would be very slight for values of $a$ in the neighbourhood of the critical value $a=\pi / \sqrt{2}$. This can be seen from the results, derived by numerical integration, given in Table 2 .

TABLE 2

Dependence of the ratio $\Delta$ of internally generated heat to the average heat flux on the horizontal wave number $a$, for a given value of the Rayleigh number $R=1.121 \times 10^{8}$

\begin{tabular}{|c|c|c|c|}
\hline$a$ & $\Delta$ & $a$ & $\Delta$ \\
\hline 0.0222 & 0.2187 & 2.221 & 0.2482 \\
\hline 0.2221 & 0.2435 & 11.11 & 0.2484 \\
\hline 0.4443 & 0.2459 & 17.77 & 0.2483 \\
\hline 1.111 & 0.2476 & 24.44 & 0.2483 \\
\hline
\end{tabular}

\section{Conclusions}

It has been shown in the previous pages how it is possibe to estimate the effects of viscous dissipation on non-linear convection at high Rayleigh 
number with the help of asymptotic methods. The analysis has been confined to the case where the Boussinesq approximation holds and for rolls and square and rectangular planforms of the convection cells. The amount of asymmetry in the vertical velocity and average temperature distributions has been estimated and an accurate expression derived for the ratio of internally generated heat to the average heat flux through the layer.

If the thickness of the layer is of the order of the temperature scale height the Boussinesq approximation is no longer valid and one has to make use of the anelastic approximation. Such a procedure would have to be undertaken for instance in a study of the role of viscous dissipation in deep convection, associated with supergranulation, in the outer layers of the sun. Recently a start has been made with the investigation of non-linear convection in a compressible medium using the anelastic approximation (Van der Borght [7]) and it is hoped to extend this work to inclure the effects of viscous dissipation in the near future.

\section{References}

[1] J. M. Hewitt, D. P. McKenzie and N. O. Weiss, 'Dissipative heating in convective flows', $I$. Fluid Mech. 68 (1975), 721.

[2] L. N. Howard, 'Notes from summer program in geophysical fluid dynamics', Woodshole Oceanographic Inst., 65-51, Vol. 1 (1965), 125.

[3] P. Ledoux, M. Schwarzchild and E. A. Spiegel, 'On the spectrum of turbulent convection', Ap. J. 133 (1961), 184.

[4] D. L. Turcotte, A. T. Hsui, K. E. Torrance and G. Schubert, 'Influence of viscous dissipation on Bénard convection', J. Fluid Mech. 64 (1974), 369.

[5] R. Van der Borght, 'Finite amplitude convection in a compressible medium', Publ. Astr. Soc. Japan 23 (1971), 539.

[6] R. Van der Borght and J. O. Murphy, 'The combined effect of rotation and magnetic field on finite amplitude thermal convection', Austr. J. Phys. 26 (1973), 617.

[7] R. Van der Borght, 'Finite amplitude convection in a compressible layer with polytropic structure', Austr. J. Phys. 28 (1975), 437.

[8] B. E. Waters, Ph.D. Thesis, Monash University (1971).

Department of Mathematics,

Monash University,

Clayton, Vic., 3168,

Australia. 